This item was submitted to Loughborough's Research Repository by the author.

Items in Figshare are protected by copyright, with all rights reserved, unless otherwise indicated.

\title{
Transient computational fluid dynamics modelling of the melting process in thermal bonding of porous fibrous media
}

PLEASE CITE THE PUBLISHED VERSION

http://dx.doi.org/10.1177/0954408912462184

\section{PUBLISHER}

(C) IMechE. Published by Sage.

\section{VERSION}

AM (Accepted Manuscript)

\section{PUBLISHER STATEMENT}

This work is made available according to the conditions of the Creative Commons Attribution-NonCommercialNoDerivatives 4.0 International (CC BY-NC-ND 4.0) licence. Full details of this licence are available at: https://creativecommons.org/licenses/by-nc-nd/4.0/

\section{LICENCE}

CC BY-NC-ND 4.0

\section{REPOSITORY RECORD}

Peksen, Murat, Memis Acar, and W. Malalasekera. 2019. "Transient Computational Fluid Dynamics Modelling of the Melting Process in Thermal Bonding of Porous Fibrous Media”. figshare. https://hdl.handle.net/2134/25689. 


\title{
Transient computational fluid dynamics modelling of the melting process in thermal bonding of porous fibrous media
}

\author{
M. Peksen ${ }^{2}$, M. Acar ${ }^{1}$ and W. Malalasekera ${ }^{1}$

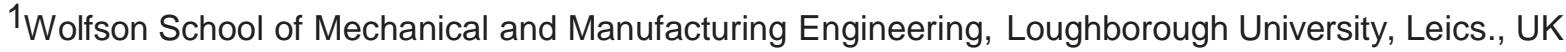 \\ 2 Institute of Energy and Climate Research (IEK), Fuel Cells, Modelling and Simulation Research Group, \\ Forschungszentrum Jülich $\mathrm{GmbH}$, Jülich, Germany
}

\begin{abstract}
A continuum model of the melting process in porous fibrous media is introduced. The fluid flow, heat transfer and phase change within the porous nonwoven web is numerically solved using computational fluid dynamics. Boundary conditions from an experimentally validated whole system model of a typical industrial machine, producing fibrous webs are incorporated. The presented model shows the capability to investigate the phase change during heating of the thermoplastic fibres during nonwoven web formation. Moreover, the fibres' geometrical information and constitutive equations, describing the material behaviour are included. The approach considers the fibre thickness, sheath fraction, and thermophysical properties like melting temperature, latent heat of fusion and the liquid fraction, enabling the assessment of different fibre types and to determine the properties of the fabric. The model results reveal that the web porosity has the most significant effect on the melting process among the considered parameters. Thermal gradients that occur inside the web are due to the combined convection and latent heat of fusion effect, which stores heat to melt the fibres. The model is applicable to a wide variety of systems ranging from textiles, fibrous beds, ceramics, membranes and porous composite materials.
\end{abstract}

Keywords

Computational fluid dynamics, porous media, nonwoven, phase change, thermal bonding, thermoplastic fibres

\section{Introduction}

Porous media theories play an important rolein many branches of engineering, including material science, chemical engineering, soil mechanics, biomechanics as well as membrane and textile technology. Porous materials are encountered literally everywhere in everyday life, in technology and in nature. For the purpose of this study, a solid containing holes or voids, either connected or non-connected, dispersed within it in either regular or random manner will be classified as a porous material provided that such holes occur relatively frequent within the solid. ${ }^{1}$ Most of the approaches regarding the porous media applied in this research are equally applicable to a wide variety of systems ranging from textiles, fibrous beds, ceramics, membranes and porous composite materials. Flow phenomena discussed in the present research is for static porous media, i.e. deformable media, as well as those whose morphology change due to chemical reaction are not considered. The scope and importance of transport phenomena in porous media are emphasized in this research; therefore, a fluid can flow through a porous material only if at least some of the pores are interconnected.
This, in this case, refers to a permeable porous material like the nonwovens. Nonwovens are a sheet, web, or batt of natural or man-made fibres or filaments, excluding paper, that have not been converted into yarns, and that are bonded to each other by any of several means. ${ }^{2}$ These include mechanical, chemical, thermally; especially, through hot convective air, as it is considered for this present study. Some of the import- ant areas where nonwovens used are geo-textiles, filtration, membrane technologies, and materials for building, thermal and sound insulating, and aerospace.

During manufacturing, most of the laid loose webs have an insufficient strength as formed, and require an additional bonding procedure, in order to provide the produced nonwoven with its intended properties. The mechanical properties such as tensile strength and bending properties of nonwoven webs exceedingly depend on the degree of bonding. To achieve the desired properties of the nonwoven webs, the bonding process is therefore, the most important part during production. 
One of the bonding techniques used is to guide hot air through the unbonded permeable non- woven web, usually wrapped around a perforated rotating drum. The method enables a rapid heat transfer, so the temperature of the fibres can be controlled accurately, which permits softening or melting of the lower melting polymer while protecting the higher melting fibres from thermal damage. An illustration of a through-air bonding process is presented in Figure 1.

Uniform temperature and airflow are paramount for the nonwoven product quality. However, to set the optimum heating and bonding conditions for the through-air bonding is difficult to establish. The nonwoven fabrics will be weak if there is insufficient bonding; on the other hand, the fibres will melt and are subjected to plastic deformation if excessive heat is applied. Details of an industrial thermal bonding machine and the bonding process are given by Peksen et al. ${ }^{3,4}$ The nonwovens used in this research are produced from continuous filament fibres; the fibres are bi-component spun fibres that have two different polymer phases in their cross-section. Each phase has its own distinct properties and contributes to a particular property of the fibre that would not be provided without the presence of the phase. Figure 2 demonstrates the photomicrograph of the used sheath-core fibre with circular sheath, shut inside a S200 type Colback ${ }^{\circledR}$ nonwoven fabric.

In the cross section of these fibres PA6 polymer forms an outer sheath phase around the inner core made of PET of the second phase.
The topic solid- liquid phase change in porous media has received attention of several researchers because of the process not only occurs in natural world such as natural seasonal freezing/thawing in cold regions, but also has practical applications in material engineering and thermal energy storage ${ }^{5-7}$ In these studies, the porous solid did not participate in the phase change phenomenon. Beckermann and Viskanta ${ }^{8}$ have developed a model for solid-liquid phase change, considering two materials present, a fixed porous matrix, and a phase change material that existed in either the solid or the liquid state.

In a previous study, Hossain et al. ${ }^{9}$ presented a comprehensive CFD study considering the thermofluid flow through a porous web and the parameters affecting its bonding. The bonding of individual fibres was also combined in a micro scale. However, the thermal and flow boundary conditions considering

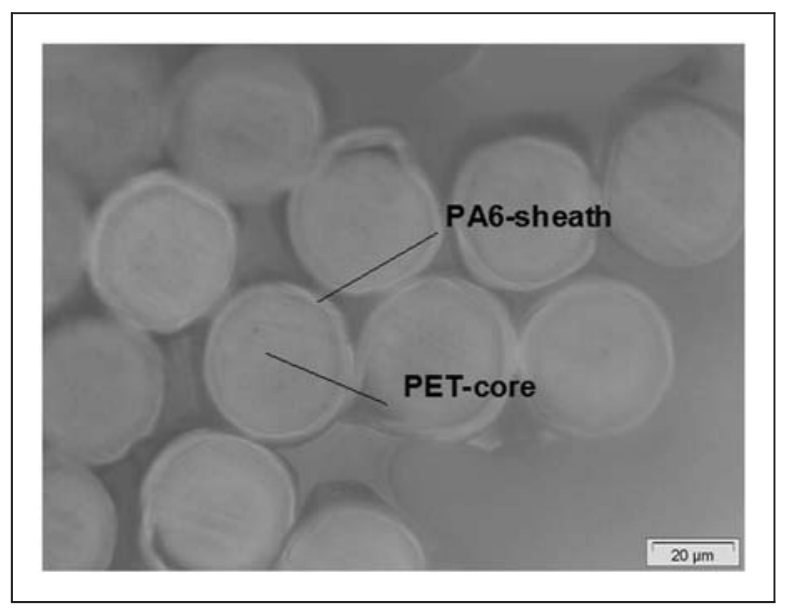

Figure 2. Sheath core fibre photomicrograph of Colback ${ }^{\circledR}$.

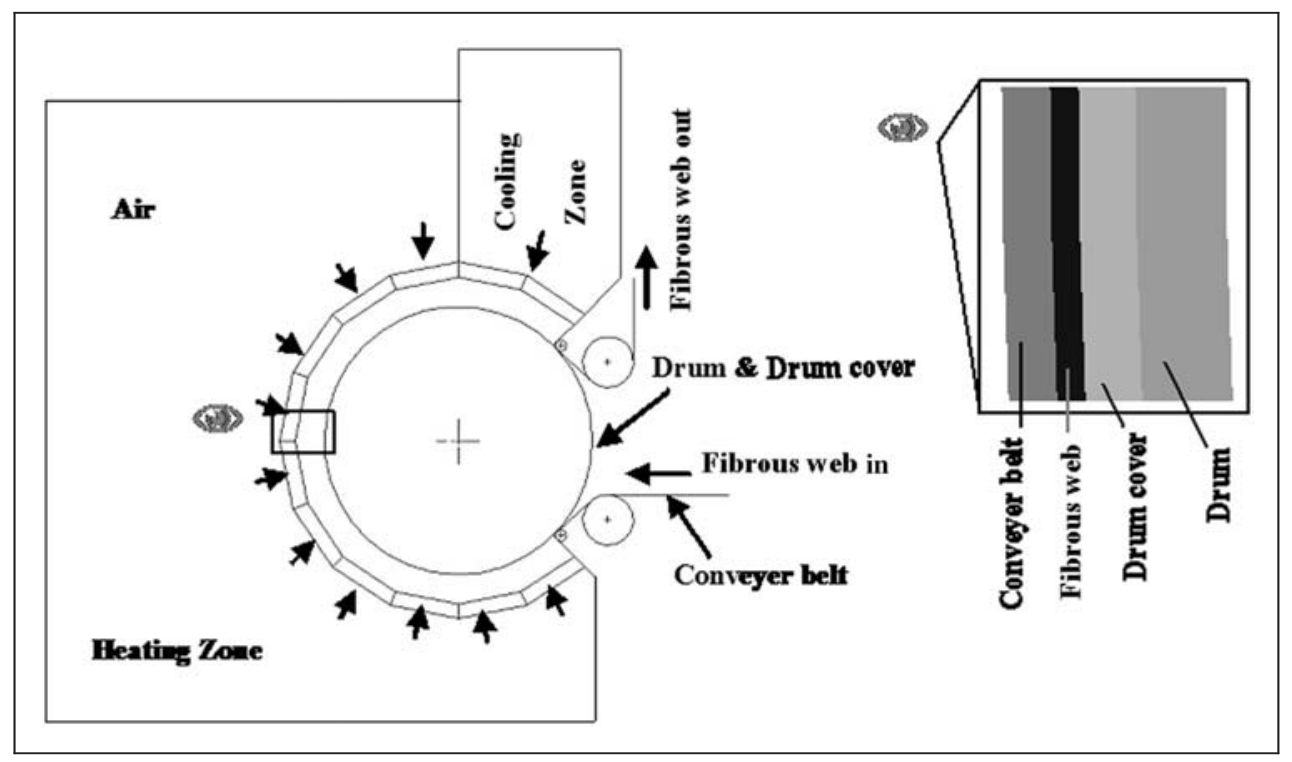

Figure 1. Schematic view of a typical thermal bonding machine and components (Peksen et al. ${ }^{4}$ ). 
typical through air bonding machine components has not been accounted. The present study introduces a complimentary product optimisation model based on computational fluid dynamics (CFD). Thermal boundary conditions from a typical industrial machine are incorporated into the model. The model considers the fibres' geometrical information and constitutive equations, describing the material behaviour. The approach considers the fibre thickness, sheath fraction, and thermophysical properties like melting temperature, latent heat of fusion and the liquid fraction, enabling the assessment of different fibre types, and to determine the properties of the fabric. A comprehensive parametric study is introduced with the aid to investigate the influence of the fibre properties on the bonding process and in particular the melting process.

\section{Mathematical modelling}

Processes related to melting and solidification encompasses a range of engineering and scientific disciplines and occurs in many applications among which the thermal bonding process of porous nonwovens is one. During the bonding process, to achieve the desired thermofusion of the bicomponent fibres, melting and solidification takes place. The numerical modelling of phase change problems are generally divided in two groups.

The first group utilises independent conservation equations for each phase and couples them with appropriate boundary conditions at the phase interface. Such methods are often referred to as multiple region or multiple domain solutions. The second group consists of single region (continuum) formulations, which eliminates the need for separate phase conservation equations. ${ }^{10}$ The multiple region approach requires the formulation of the discrete interfaces between the phases, which complicates the mathematical description for the porous fabric extremely. Unlike pure substances, multi constituent systems do not exhibit a sharp interface between solid and liquid phases. In fact, due to impurities (intentional or otherwise), discrete phase change rarely occurs in practice.

Since the complicated phase interfaces are not tracked, single region formulation for the melting of the nonwoven fabric layer is suitable for treating the continuous transition between the solid and liquid phases. In order to account for the interfacial structures of the constituents, the macroscopic model equations are based on volumetric averaging of the microscopic conservation equations. To enable the treatment of the entire domain as a single region governed y one set of conservation equations, the energy -model of Beckermann and Viskanta ${ }^{8}$ and Hossain et al. ${ }^{9}$ is used. For the averaged mass and momentum conservations as well as the derivation of the flow resistance terms in the porous media, it is referred to Peksen et al. ${ }^{4,11,12}$ and will not be repeated here. The volumetrically averaged macroscopic transport equations with phase change, assume to occur volumetric- ally over a small temperature range. The porous media has been treated as a homogenous, isotropic, multicomponent material with an initial solidus temperature. Moreover, the porous matrix and the solid region are non-deformable and each state has constant thermophysical properties.

The liquid fraction of the sheath fibre $y$ is in the melt region defined as completely liquid, so that the liquid sheath fibre volume fraction $y$ is equal to the sheath volume fraction ", which is "(1-') of the air volume fraction (porosity) ' of the volume and is 1. In the solid region, $1 / 4 y^{1 / 4} 0$. Due to the porous matrix irregularities, the volume element close to the melting temperature might be simultaneously in the liquid and solid phase. In this situation the liquid fraction is 05 y 51 ; and $0<y<$ "(1-'). The averaged energy equation for the matrix/solid/liquid mixture can be written as

$$
\begin{aligned}
& \frac{\partial}{\partial t}\left[\varphi \rho_{a} h a+(1-\varphi)\left(\varepsilon\left(\gamma \rho_{l} h l+(1-\gamma) \rho_{s} h s\right)\right.\right. \\
& \left.\left.\quad+(1-\varepsilon) \rho_{c} h c\right)\right]+\nabla \cdot\left(\rho_{a} h a \vec{u}\right)=\nabla \cdot\left(k_{e f f} \nabla T\right)
\end{aligned}
$$

$h_{\varphi}$ refers to the enthalpy of the constituents in which $a$ refers to the air; for the melting sheath fibre component $l$ is the liquid state, $s$ the solid state and $c$ represents the core part of the bi-component fibre, which is due to its thermophysical properties in solid state. $k_{e f f}$ and $T$ are the effective thermal conductivity and temperature of the mixture respectively. Even though the phase change is assumed to occur at a discrete temperature, in a volume element containing the porous matrix as well as the solid and liquid, the average temperature may be slightly higher or lower than the melting temperature.

Hence, it is assumed that both solid and liquid may exist simultaneously in a volume element, if its temperature lies within a small temperature difference $\Delta T$ on either side of the fusion temperature. For a volume element undergoing phase change (i.e. $0<y<1$ ), a change in the mean enthalpy of the fluid is due to a change in the sensible heat of the liquid/solid mixture plus the contribution of the latent heat, which can be expressed as

$$
\begin{aligned}
& d\left[\gamma \rho_{l} h_{l}+(1-\gamma) \rho_{s} h_{s}\right] \\
& \quad=\left[\gamma \rho_{l} c_{l}+(1-\gamma) \rho_{s} c_{s}\right] \mathrm{d} T+\rho_{l} \Delta h d \gamma
\end{aligned}
$$

where $c$, is the specific heat capacity of constituent. Substituting equation (2) into equation (1) and simplifying leads to the conservation equation of energy for melting of liquid saturated porous media.

$$
\begin{aligned}
& \overline{\rho c} \frac{\partial T}{\partial t}+\rho_{a} c_{a}(\bar{u} \cdot \nabla T) \\
& \quad=\nabla \cdot\left(k_{e f f} \nabla T\right)-(1-\varphi) \varepsilon \rho_{l} \Delta h \frac{\partial \gamma}{\partial t}
\end{aligned}
$$


The equation is the volume averaged energy equation for the general control volume containing the porous medium solid-liquid mixture. The mean thermal capacitance of the solid-liquid mixture is defined as

$$
\begin{aligned}
\overline{\rho c}= & \varphi \rho_{a} c a+(1-\varphi) \\
& \times\left[\varepsilon\left(\gamma \rho_{l} c l+(1-\gamma) \rho_{s} c s\right)+(1-\varepsilon) \rho_{c} c c\right]
\end{aligned}
$$

The effective thermal conductivity $k_{e f f}$ of the mixture is calculated using the continuum theory of mixtures considering the volume fraction of the bicomponent fibres and can be expressed as

$$
k_{e f f}=\varphi k a+(1-\varphi)[\varepsilon(\gamma k l+(1-\gamma) k s)+(1-\varepsilon) k c]
$$

The thermal conductivity of the liquid saturated nonwoven under static conditions is assumed to be the same as that for a transient system with a flowing fluid in which dispersion effects may be present. Although in solidification systems, it is important to recognise the large differences in scales upon which the various phenomena occur. The development of the transport equations takes place on the macroscopic scale with a number of computational cells being used to resolve the two-phase region. ${ }^{13}$

Despite the melting of the fibres in the continuum nonwoven is investigated on a macro scale, the process is considered to be a meso model due to the problem level located between the four layer through-air bonding system and the discrete fibres. The attempted approach considers the fibre thickness, sheath fraction, and thermophysical properties like melting temperature, latent heat of fusion and the liquid fraction, enabling the assessment of different fibre types, and to determine the properties of the porous fabric. The implementation of the physics and mathematical description into the model are done using user-written macros.

\section{Computational modelling}

From a more fundamental point of view, it is of interest to investigate the phase change and melting process of the nonwoven fabric during the bonding process. The approach is based on the results achieved from the CFD analyses performed in 2D for the whole bonding process, and considering all four machine components i.e. drum, drum cover, conveyer belt and the nonwoven web. ${ }^{3,4}$ The experimentally validated CFD results from the used model were incorporated as boundary conditions to the presented model. This will enable to investigate the phase change of the web during the thermofluid flow through the whole machine components, and not assuming constant heat flux or flow conditions on the top and bottom borders of the web. Moreover, as the model is in particular useful for the product optimisation, it is an efficient way to investigate the nonwoven layer in detail separately. The model considers the fibres' geometrical information and constitutive equations describing the material behaviour. These are implemented in a single model due to the computational effort required to solve the macros rather than incorporating within the macro model developed and introduced for rapid process optimisations. ${ }^{3,4}$ Figure 3 demonstrates the adopted approach.

The transient thermofluid flow analysis through the whole bonding machine over time is performed. The result set of the analysis is transferred to the nonwoven layer model, considering the thermal and flow information obtained from the conveyer belt and drum cover sandwiching the web. This information is applied to the boundaries of the used 2D continuum model. The mathematical model is applied to the porous web geometry using user defined macros based on $C$ programming language. The commercial CFD code FLUENT has been employed. During the analysis, it is assumed that the liquid fraction of the nonwoven layer is flowing through the solid matrix. The porosity is not considered as constant but modelled as pseudo porous media where it is a function of the representative latent heat content of each computational cell. As the latent heat approaches zero, the porosity will approach zero and the source terms due to Darcy ${ }^{14}$ and Forchheimer terms ${ }^{15}$ will dominate the momentum equation, and force the related velocities being close to zero.

As the latent heat tends to its maximum, the porosity approaches one. The appropriate function to operate between these two limits is chosen to be a direct proportionality, occurring between the latent heat and the fraction of solid melted. In order to establish a phase change, the latent heat contribution is specified as a function of temperature and is associated with the liquid fraction in the so called mushy zone. The porosity takes the values, 1 in the liquid phase, 0 in the solid phase, and $0<e<1$ in the mushy zone.

\section{Results and discussion}

CFD analyses were performed using the introduced model. Figure 4 illustrates the contour plots of the liquid fraction over time with its temperature distribution within the nonwoven fabric. The simulation results obtained from a previous study ${ }^{4}$ that considers the whole system components have been used. The results are based on the whole system analysis that was performed at $225^{\circ} \mathrm{C}$ air inlet temperature and $0.665 \mathrm{~m} / \mathrm{s}$ air velocity. The temperature and velocity distribution resulting for the upper and bottom web borders, neighbouring the drum cover and the conveyer belt (as the porous web is in reality sandwiched between these components) are implemented as initial and boundary conditions within the present analyses. The initial porosity of the considered web has been 0.88 . Presented results are scaled as to enable accurate comparison between the plots. 


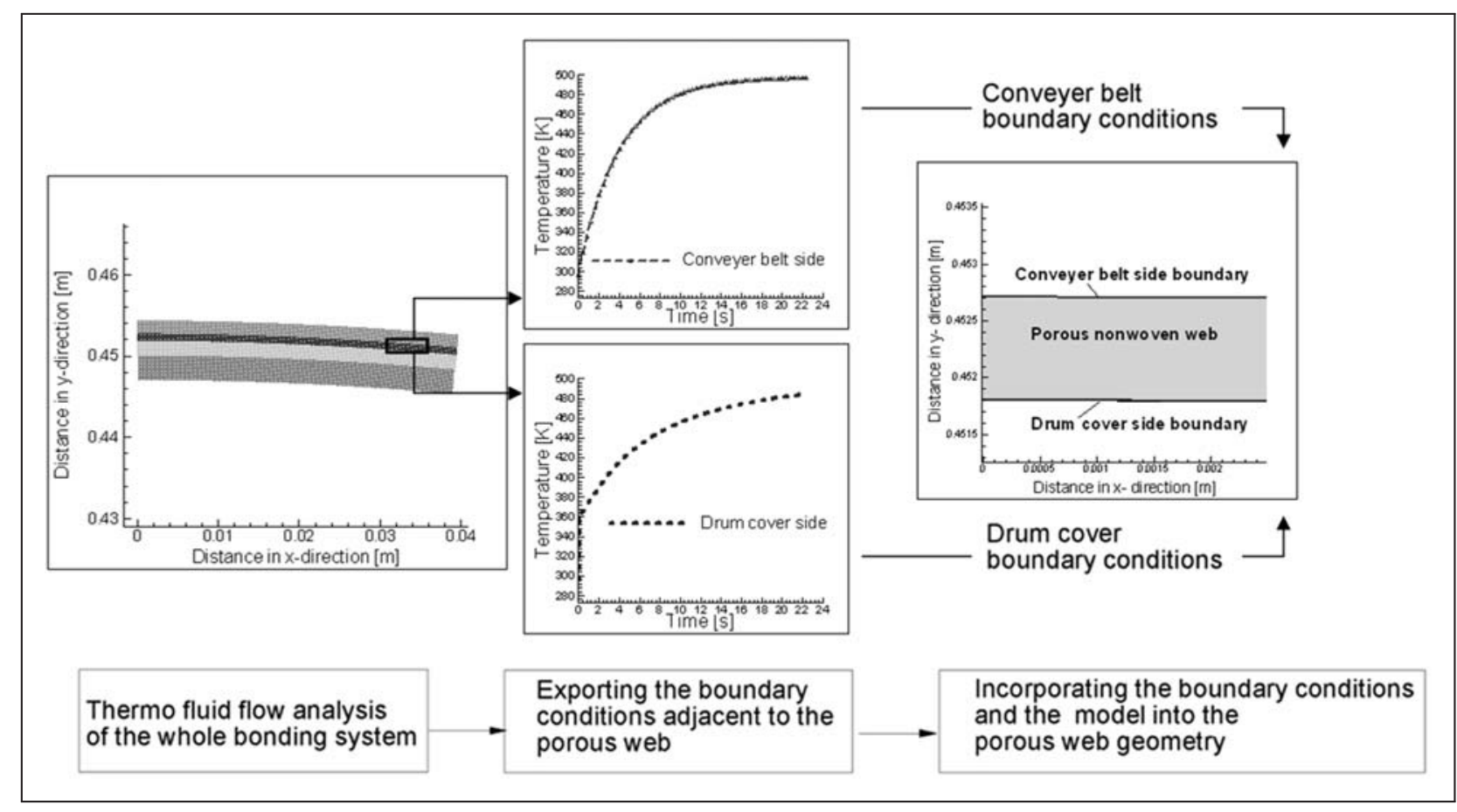

Figure 3. Employed computational model and the applied boundary conditions from the macro model.

The predicted results show that the liquid fraction is zero i.e. completely solid state at 10 seconds. The temperature is still increasing and a gradient with in the nonwoven is visible. The sheath fibre is still in solid state at this time. The phase change process between the time intervals of $14.1 \mathrm{~s}$ to $15 \mathrm{~s}$ reveal that at $14.1 \mathrm{~s}$ the sheath fibre i.e. the nylon layer of the bi-component fibre is no more in total solid state. Due to the porous matrix irregularities, the occupied volume is simultaneously in the liquid and solid phase, that is, the liquid fraction of the sheath fibre $y$ and the sheath volume fraction related to the air volume fraction (') of the volume remain between zero and one. Therefore, the latent heat which is defined as a function of temperature is no more zero. This is because the temperature at this period is slightly approaching the melting temperature. The latent heat is associated with the liquid fraction; hence, the liquid fraction is also no more zero. Under the given process conditions the introduced essential part of the model is developing. The volume simultaneously occupied by the liquid and solid should theoretically, be at the fusion temperature i.e. the melting temperature. At this temperature the difference between the liquid and solid enthalpies is equal to the latent heat of fusion.

Since the phases are assumed to be in local thermal equilibrium this temperature would be expected. However, in reality the mean temperature may be slightly above the fusion temperature, although the local temperature at the solid-liquid interface is equal to the melting temperature. A similar case applies if the volume is almost completely solid, which is the case for this time interval, the temperature can be slightly below the fusion-melting temperature.

This violates the local thermal equilibrium assumption, but because of the finite gradients existing within the nonwoven fabric it is a good approximation. The research considers that if the mean temperature is within a small interval $2 \mathrm{f} . . T$, around the fusion temperature, the simultaneous existence of solid and liquid within the volume may occur. The mean temperature is below the fusion temperature hence, the liquid fraction is below 0.5 . The development of the mushy region for time, the time interval $15-22.4 \mathrm{~s}$ is shown in Figure 5.

Note that the liquid fraction $y$, is assumed to be equal to 0.5 for $T^{1 / 4} T_{m}$ i.e. in equality of the mean temperature and the fusion temperature. This is due to the chosen linear relationship between the liquid fraction and the mean temperature. As the imposed temperature approaches the fusion temperature, heat is absorbed for the softening process. The plots show clearly how the temperature and the liquid fraction is rising over time. At time $15 \mathrm{~s}$ a small liquid fraction is observed, where as this value exceeds the fusion temperature at time $20 \mathrm{~s}$.

Finally the temperature gradient reduces at time of $22.4 \mathrm{~s}$, resulting in the highest liquid fraction values. To compare the mushy region results thoroughly, the start of converting from a solely solid state to liquidsolid mixture, the time the fusion temperature is reached with the liquid fraction $y$ as 0.5 , and the end of the heating zone is observed on the same scale. The results are illustrated in Figure 6. 


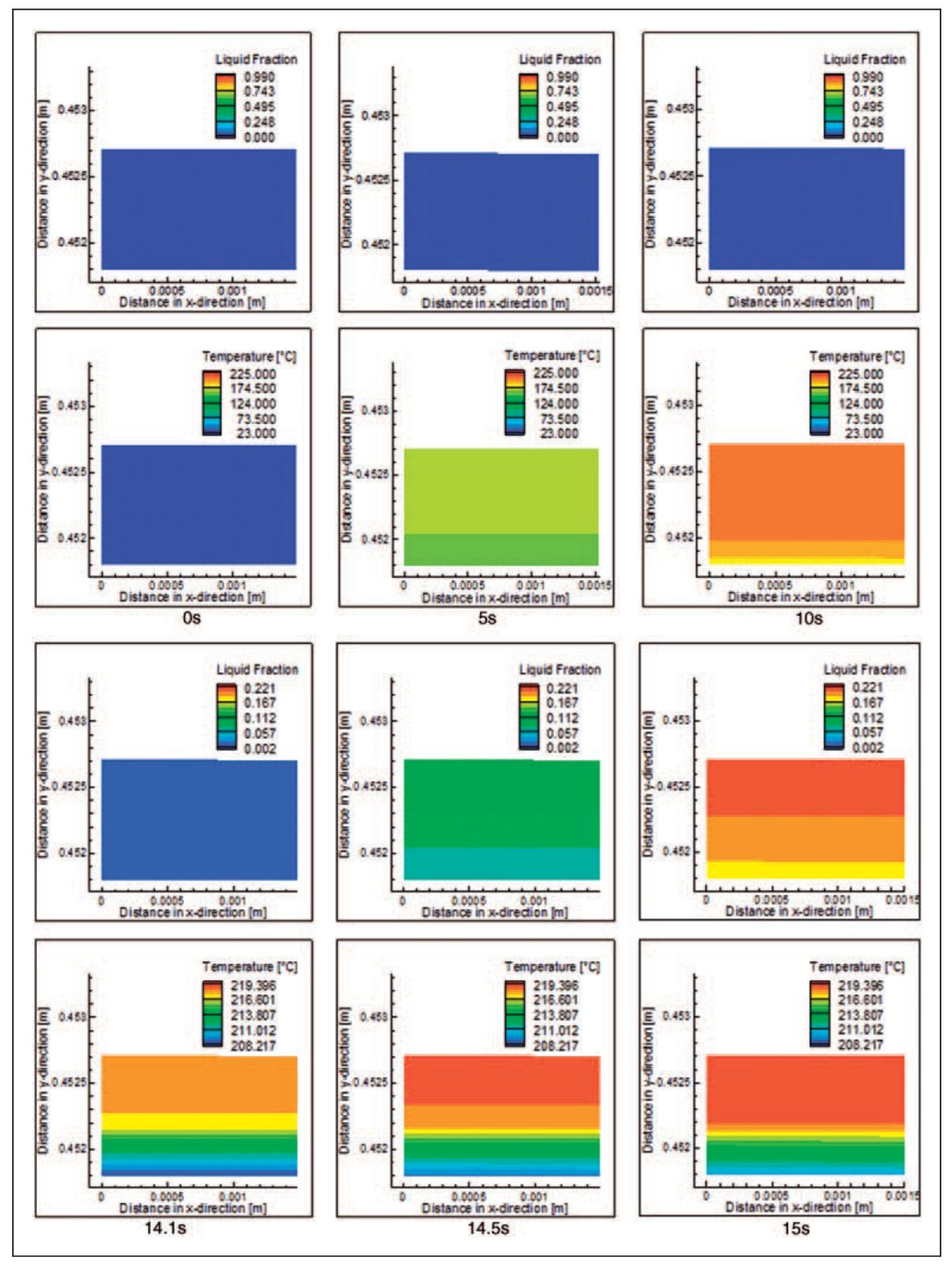

Figure 4. Liquid fraction and temperature contour plots of nonwoven fabric for time interval 0-15s.

The results illustrate the time evolution of the melting process; the solid lines represent the numerical predictions of the contour lines. The mushy region starts at 14.1 and reaches the 0.5 value, which is specified as the melt fraction at the fusion temperature at $16.7 \mathrm{~s}$ of the heating process. The increase in temperature can be seen developing in a direction from the top to the bottom of the plot which is the direction of the hot air flow. It can be seen that for heating at $14.1 \mathrm{~s}$ and $16.7 \mathrm{~s}$ the melting front is almost parallel to the hot upper and lower surfaces indicating diffusion dominated melting process. The temperature plots also confirm this as illustrated. It is observed that as the temperature gradient within the solid region was at $t^{1 / 4} 14.1 \mathrm{~s}$ high, the melting process was low.

The melt front exhibits a shape typical for convection dominated melting at $22.4 \mathrm{~s}$. The interface moves faster and the contour lines are distorted. This is due to the latent heat convection source term. The flow direction away from the bottom part increases the heat loss and hence extends the mushy region. At the same time the heat from the bottom part, retards the growth of the mushy region making the slight bulge in the region. 


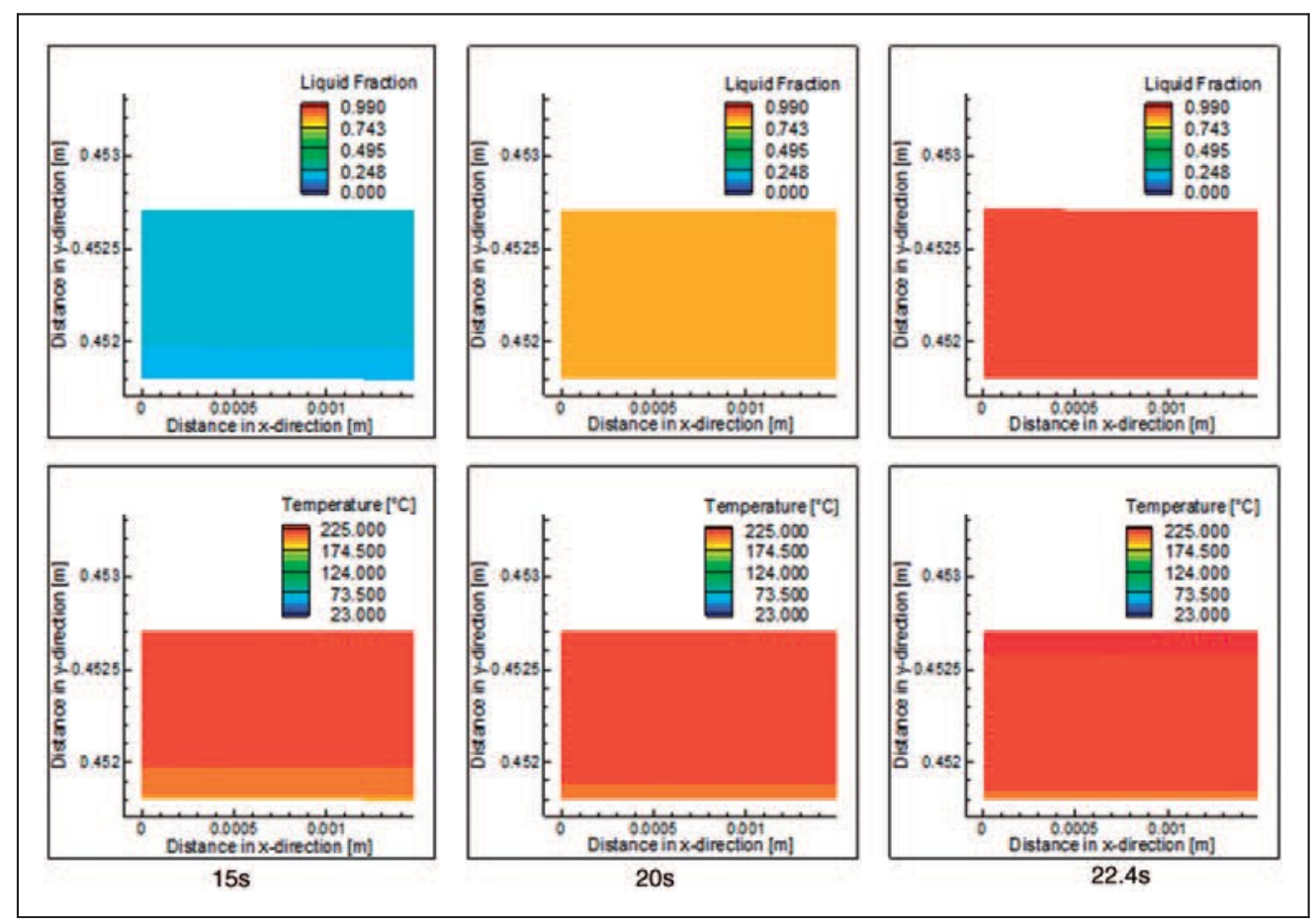

Figure 5. Liquid fraction and temperature contour plots of nonwoven fabric for time interval 15-22.4s.

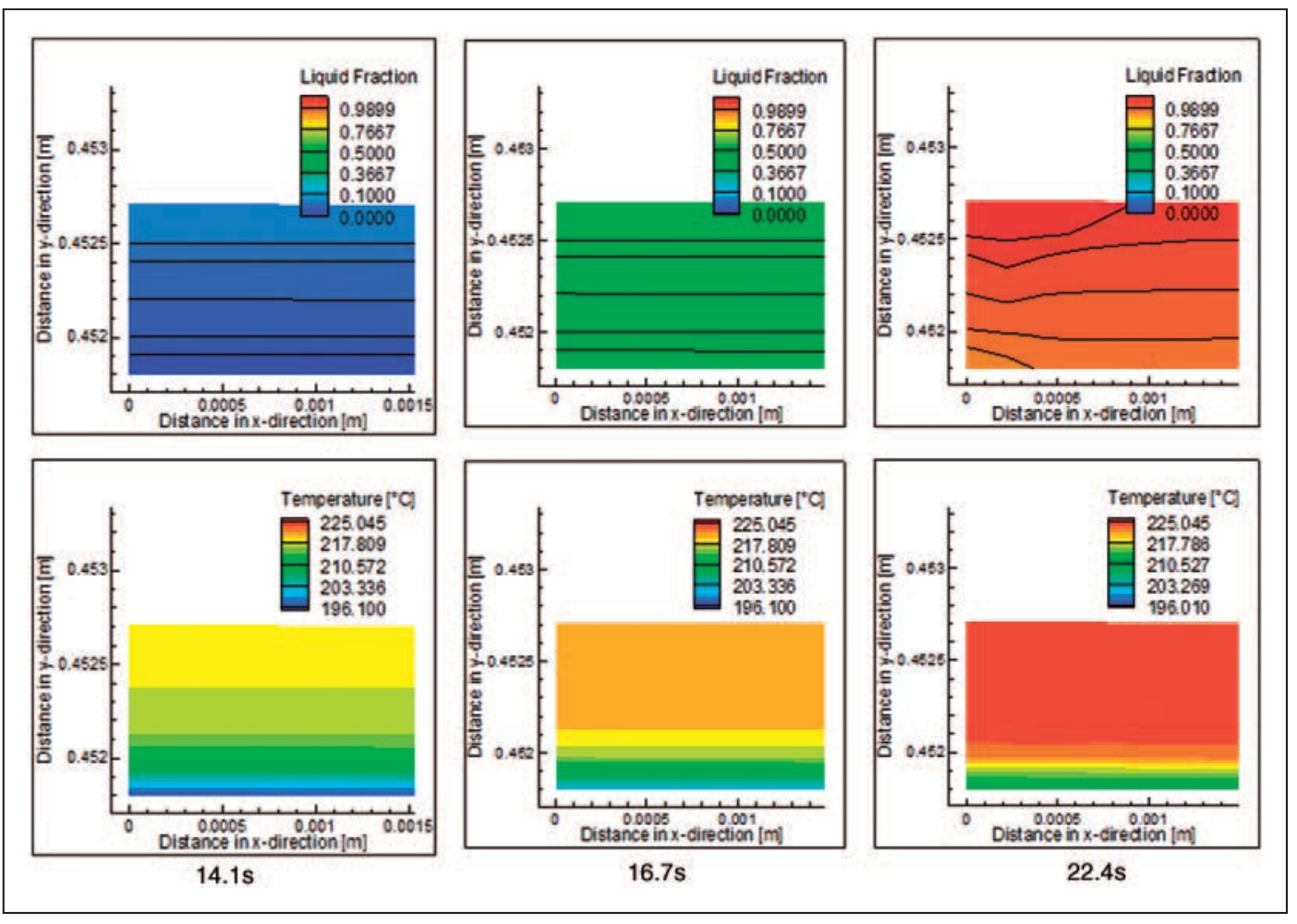

Figure 6. Liquid fraction with contour lines and temperature contour plots of nonwoven fabric for time interval 14.I-22.4s.

\section{Effect of fibre and web properties on the bonding process}

In order to investigate the influence of the fibre properties on the bonding process and in particular the melting process, the developed model is used. For the CFD analyses, bi-component fibres that have two different polymer phases in their cross-section are considered. The sheath-core fibres are investigated in terms of fibre diameter, sheath fraction and the porosity of the fabric. 


\section{Fibre diameter}

The fibre diameters used in this study are 31.71, 31.30 and $15.15 \mathrm{~mm}$, respectively. The first two fibre diameters are used in Colback ${ }^{\circledR}$ production, and the last one is used by KoSA. ${ }^{8}$ The results presented in Figure 4 considered bi-component fibres with a diameter of $31.71 \mathrm{~mm}$. According to these results it is apparent that the fibre reached the melting fraction 0.5 , i.e. the melting temperature of $221^{\circ} \mathrm{C}$ after approximately $17 \mathrm{~s}$. Considering this as a comparison starting point, the simulation results at this time step were examined in more detail. Radial profiles of the temperature and melt fraction for the nonwoven web with porosity of 0.88 are compared. Figure 7 gives the simulation results of the temperature distribution inside the nonwoven web and the corresponding distribution of melt fractions of the fibres.

The adjacent boundaries of the porous web are denoted as belt and drum cover, indicating the top and bottom sides of the nonwoven web. The predicted results show that the effect of the fibre diameter has no influence on the temperature profile as well as on the melt fraction of the used bi-component fibres. It should be noted that the sheath fraction in all three cases is kept constant as 0.273 .

\section{Fibre sheath fraction}

The sheath fraction of bi-component fibres is defined as the ratio of the sheath fibre cross-sectional area to the total fibre area. To investigate the effect of the sheath fraction, two different values are considered: 0.273 and 0.4861 . These values correspond to real products used by COLBOND and KoSA fibres, respectively. CFD analyses are performed and the predicted results are presented in Figure 8.
The results show that the temperature profiles for the given boundary conditions is as expected the same, whereas the fibre with the higher sheath fraction causes a decrease in melt fraction. This is due to the higher amount of mass, which needs to absorb more energy to result in the same amount of melt fraction. Though this difference is not a significant amount it will have an effect of the number of bonding points formed between the fibres. This on the other hand would result in different mechanical properties of the product. The increased amount of sheath might lead to a deeper level of bond though the fraction is slightly lower, but because of the reduced amount of the core fraction, the mechanical properties would be more affected than the difference of this melt fraction.

\section{Web porosity}

The porosity of the web is another parameter investigated using the CFD technique and the proposed model. The radial profiles of temperature and melt fraction for the porosity of $0.9,0.88,0.80$ and 0.70 are compared in Figure 9.

The temperature gradient illustrated in Figure 9(a) shows that higher temperatures are achieved at higher porosities. The temperature values are higher for higher porosities due to the higher free path of air flowing through the fabric, as well as the lower amount of mass to be heated.

This corresponds well with the melt fraction data illustrated in Figure 9(b). As it is clearly seen the melt fraction is higher when the porosity is higher. Hence the thermal gradient differences occur due to the combined convection and latent heat of fusion effect which stores heat to melt the fibres, thus lower temperatures are reached for higher porosity values within the nonwoven in comparison to the nonwovens
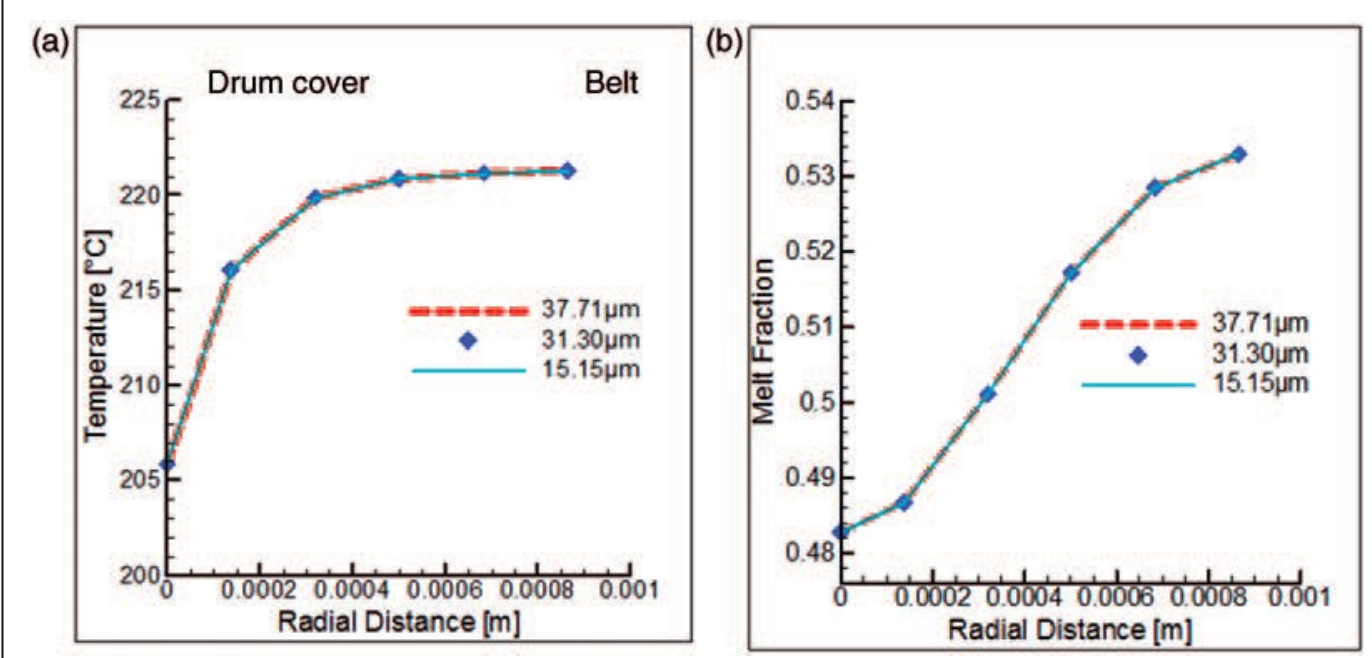

Figure 7. Web radial profile at time $t^{1 / 4} 17 \mathrm{~s}$ as a function of fibre diameter: (a) temperature profile; (b) melt fraction. 


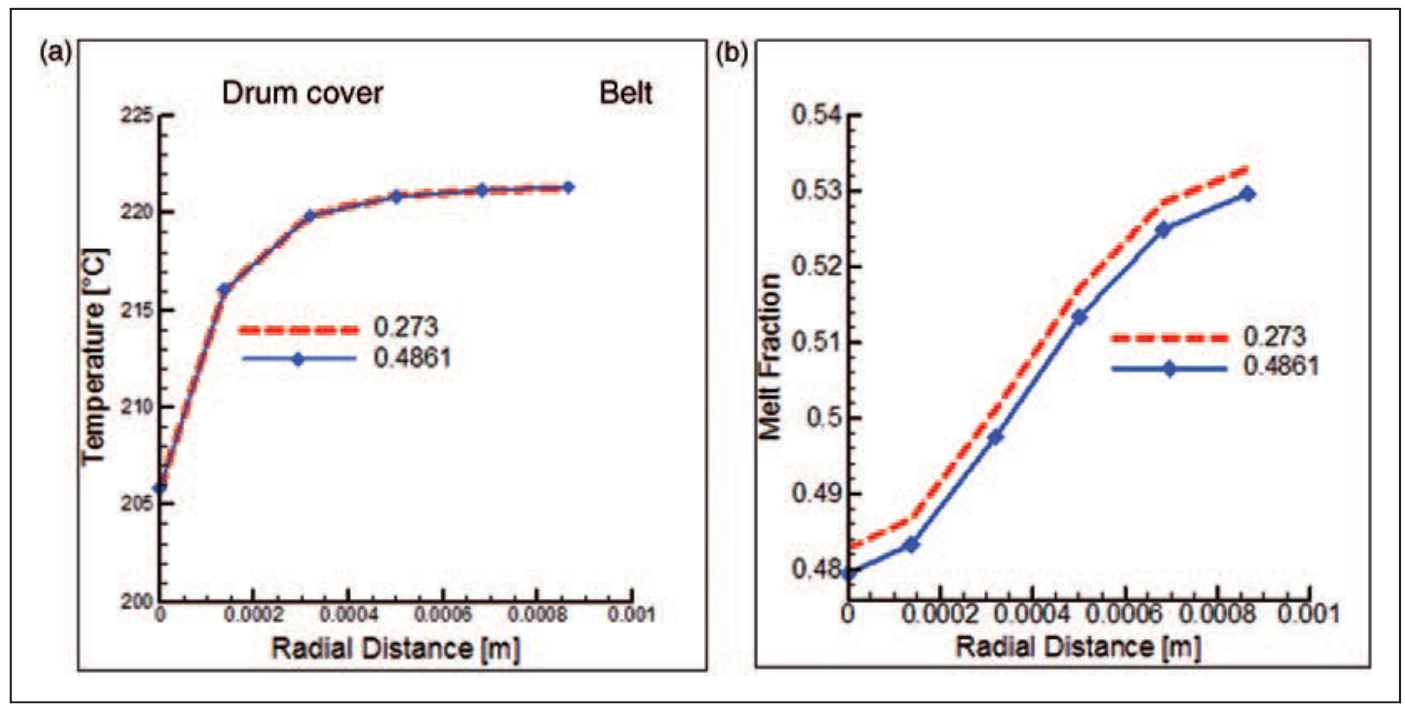

Figure 8. Web radial profile at time $t^{1 / 4} 17 \mathrm{~s}$ as a function of sheath fraction: (a) temperature profile; (b) melt fraction.

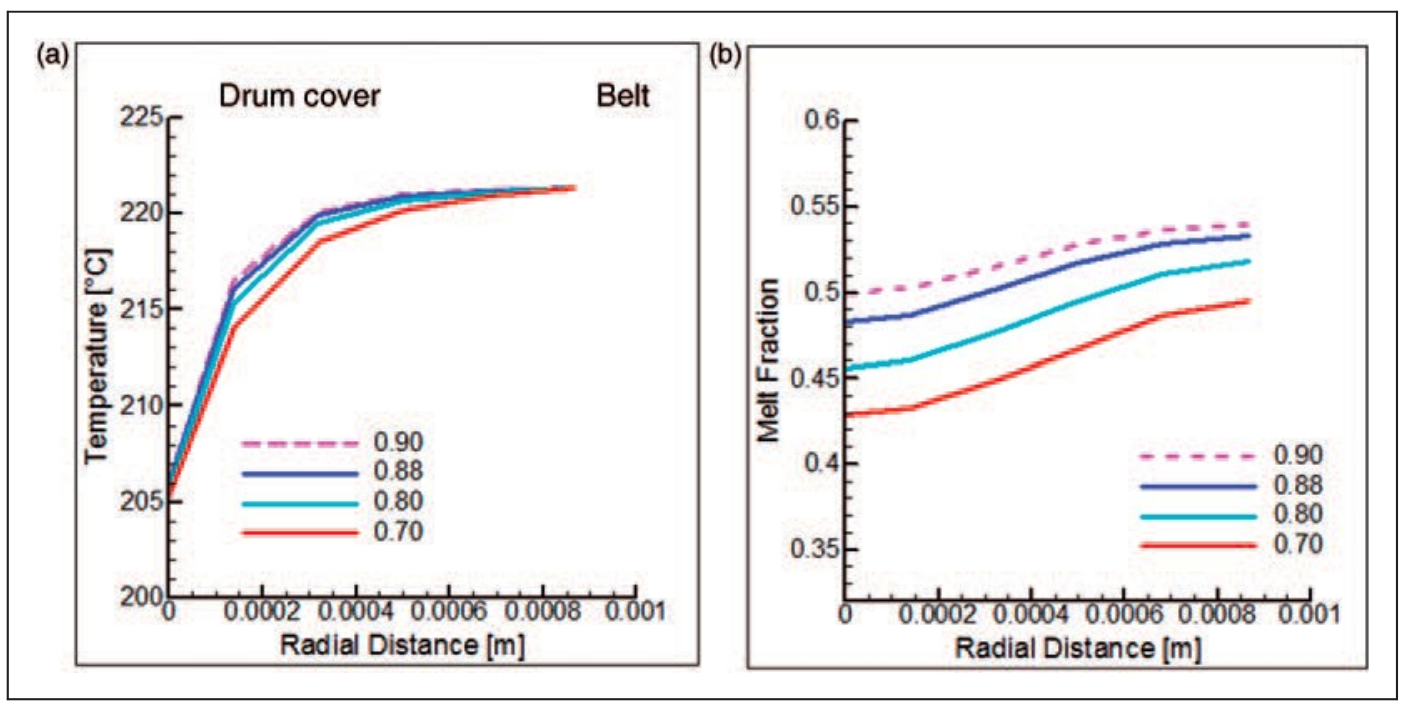

Figure 9. Web radial profile at time $t^{1 / 4} 17 \mathrm{~s}$ as a function of web porosity: (a) temperature profile; (b) melt fraction.

having a lower porosity. These results correspond to the analyses performed by Hossain et al. ${ }^{8}$ very well. From the performed parametric study, it is clearly seen that the web porosity has the most significant effect on the melting process among the considered parameters.

\section{Conclusions}

A continuum model enabling the investigation of the phase change during the heating of porous media is introduced. The approach is based on the results achieved from the CFD analyses performed for a whole thermal bonding nonwovens process, and considering all 4 machine components i.e. drum, drum cover, conveyer belt and the nonwoven web. The results from the employed model were incorporated as boundary conditions to the presented model. The model considers the fibres' geometrical information and constitutive equations describing the material behaviour. Moreover, the fibre thickness, sheath fraction, and thermophysical properties like melting temperature, latent heat of fusion and the liquid fraction enabling the assessment of different fibre types, and to determine the properties of the porous media are accounted. The model is applicable to a wide variety of systems ranging from textiles, fibrous beds, ceramics, membranes and porous composite materials, and is an excellent tool for product optimisation.

\section{Funding}

This work was supported by Colbond bv, Arnhem, The Netherlands.

\section{References}

1. Collins RE. Flow of fluids through porous materials. New York: Reinhold Publishing Corporation, 1961. 
2. Butler I. Nonwoven fabrics handbook. Cary, NC: INDA, 1999.

3. Peksen M, Acar M and Malalasekera W. Computational modelling and experimental verification of through-air bonding nonwovens process, In: Proceedings of the international nonwovens technical conference, 2007.

4. Peksen M, Acar M and Malalasekera W. Computational modelling and experimental validation of the thermal fusion bonding process in porous fibrous media. Proc IMechE, Part E: J Process Mechanical Engineering 2011; 225(3): 173-182.

5. Phanikumar MS and Mahajan RL. Non-Darcy natural convection in high porosity metal foams. Int $J$ Heat Mass Transfer 2002; 45: 3781-3793.

6. Dosanjh SS. Melt propagation in porous media. Int $J$ Heat Mass Transfer 1989; 32(7): 1373-1376.

7. Haeupl P and $\mathrm{Xu} Y$. Numerical simulation of freezing and melting in porous materials under the consideration of the coupled heat and moisture transport. $J$ Therm Envel Build Sci 2001; 25: 4-31.

8. Beckermann $C$ and Viskanta R. Natural convection solid/liquid phase change in porous media. Int $J$ Heat Mass Transfer 1988; 31(1): 35-46.

9. Hossain M, Acar M and Malalasekera W. A mathematical model for airflow and heat transfer through fibrous webs. Proc IMechE, Part E: J Process Mechanical Engineering 2005; 219: 357-366.

10. Bennon $\mathrm{W}$ and Incropera F. A continuum model for momentum, heat and species transport in binary solidliquid phase change systems - I. Model formulation. Int J Heat Mass Transfer 1987; 30(10): 2161-2170.

11. Peksen M, Peters Ro, Blum L, et al. Numerical modelling and experimental validation of a planar type prereformer in SOFC technology. Int $J$ Hydrogen Energy 2009; 34: 6425-6436.

12. Peksen M. A coupled 3D thermofluid-thermomechanical analysis of a planar type production scale SOFC

stack. Int $J$ Hydrogen Energy 2011; 36(18): 11914-11928.

13. Voller V, Brent A and Prakash C. The modelling of heat, mass and solute transport in solidification systems. Int J Heat Mass Transfer 1989; 32(9): 1719-1731.

14. Darcy HPG. Les Fontaines Publiques de la Ville de Dijon. Paris: Victor Dalmont, 1856.

15. Forchheimer P. Wasserbewegung durch Boden. Z Ver Deutsch Ing 1901; 45: 1782.

\section{Appendix}

Notation

h

H

k

$T$

a

$x$,

y

\section{Subscripts}

$a$

c

l

$S$

eff specific heat capacity $(\mathrm{J} / \mathrm{kgK})$

enthalpy $(\mathrm{J} / \mathrm{kg})$

specific energy $(\mathrm{J} / \mathrm{kg})$

thermal conductivity $(\mathrm{W} / \mathrm{m} \mathrm{K})$

temperature $(\mathrm{K})$

velocity vector $(\mathrm{m} / \mathrm{s})$

$y, z$ cartesian coordinates $(\mathrm{m})$

density $\left(\mathrm{kg} / \mathrm{m}^{3}\right)$

liquid fraction of the sheath fibre (-)

air volume fraction (-)

sheath volume fraction (-)

air

core part of the bi-component fibre

liquid state

solid state

effective

constituent 